\title{
Upper Respiratory Tract Infection Status Symptoms among the Recreational Endurance Runners- A Perspective
}

\author{
Mr. RajendarKommu* \\ Research Scholar, JNT University, Hyderabad, India.
}

*Corresponding Author: Mr. RajendarKommu, Research Scholar, JNT University, Hyderabad, India.

\begin{abstract}
Regular exercise has been recognized as an effective antidote for many diseases both of communicable and non communicable ones. People prefer to undergo different forms of exercise depending on many factors. Aerobic and anaerobic exercises are considered as the two forms of exercises that use different metabolic pathways while utilizing the different substrates. But no single exercise can be a pure aerobic or anaerobic in nature but runs in a continuum from aerobic to anaerobic. Of late, several enthusiastic persons are taking up to running as their preferred choice of fitness and this trend is increasing especially among the urban youth and urban employed, though age ranges of these recreational runners from teens to the geriatric populations. These runners who run for the sake of fitness and passion may be considered as recreational runners and most of them may be completely freshers to the exercise and sports world and may need credible and scientific preparation for gaining physiological adaptation to take up high intensity long duration runs like marathon runs. But the scenario is different one, as most of these runners are graduating themselves from running few kilometers to full marathon in few months without proper consolidation and could experience several health problems due to this quick progression. Some research studies of exercise immunology are indicating that such unadopted or less adapted high intensity very long duration aerobic efforts could induce uncontrolled oxidative and inflammatory stress causing respiratory tract infections due to tissue damage of the respiratory tracts and also due to the suppressed immunity both of mucosal and cell mediated ones. It would be ideal for the recreational runners to constantly monitor their upper respiratory infection status through credible quantitative techniques like WURSS-44 questionnaire and also monitor their lung volumes through the digital spirometer.
\end{abstract}

Keywords: High intensity aerobic exercise, oxidative stress, inflammation, immune suppression.

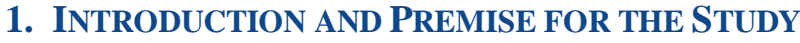

Exercise in general in any form done may improve the functional health of the individuals thereby providing enhanced immune function and disease prevention capacity ${ }^{1,18}$. Even the effect of exercise seems very favorable for both controlling and preventing both the communicable and noncommunicable diseases ${ }^{8}$. Enhanced immunity of both the mucosal and cell mediated would provide defense against the infections through the disease causing pathogens like viruses, bacteria and other organisms. While the enhanced functional health of the organism helps the individual s to better tackle the pathophysiological conditions that might provoke or trigger negative events that could enhance the non-communicable disease conditions ${ }^{10}$. This may be due to the effect of enhanced endocrinal and other possible mechanisms that might prosper for the enhanced pathological conditions. For example, exercise could induce changes in the insulin sensitivity, glucose transporter mechanisms, other cell signaling mechanisms that might alter the physiological conditions positively to tackle the metabolic syndrome conditions or else other disease prone conditions like precancerous conditions and other such conditions.

Exercise may be conducted in many forms and basing on the metabolic pathways involved with respect to the substrate utilized or the proportion of the substrates utilized for the energy to conduct the specific exercise undertaken may be divided broadly into two types. One being the aerobic exercise and the other one being the anaerobic exercise form. But, as per the exercise physiology there are no pure forms of aerobic exercise or anaerobic exercise and these two metabolic pathways are shared in proportion during the exercise program. But, when the aerobic nature exceeds anaerobic pathway in proportion the same may be considered as aerobic physical exercise. Again depending on the intensity of any exercise conducted the metabolic pathways change drastically and it happens in 
the case of elite sportspersons. In general, as the intensity of the exercise increases beyond the sixty to seventy percent of the maximum possible intensity relating to the individual, the anaerobic metabolic pathways become more dominant and this happens generally among the elite sportspersons like top class marathon runners. This may be due to the muscle preference of more toward the glycogen than towards the free fatty acids for energy derivation. Whereas, the general persons, who attempt health running in easy, light and medium intensities to derive health benefits may be more of aerobic in nature. As the more time available, the transfusion physiology of oxygen becomes more dynamic and can cause for the higher beta oxidation of fats through more availability of oxygen for energy pathways in better utilization of mitochondrial oxidative phosphorylation process. But, however, even at the higher intensity sustained aerobic running, the possibility of mitochondrial oxidative phosphorylation and beta oxidation of fats are seen in considerably high quantities.

The important considerations in understanding the negative issues with respect to the exercise seems in two aspects and they are oxidative and inflammatory aspects. As per the disease pathophysiology in general, process of uncontrolled inflammation and excessive oxidative stress could lead for suppression of immune function, may also cause for pro-cancerous conditions and such other disease prone physiological conditions. However, these two processes are in fact necessary phenomenon for initiating the antithetical process so that the body can equip enhanced functional physiology that can prevent diseases both of communicable and non-communicable. Any form of exercise, including the running initiates both the inflammation and oxidative stress on the organism and in general among the several tissues of the body simultaneously. Especially the high intensity exercises, more specifically the high intensity sustained aerobic exercises like marathon running, half marathon running, ultra endurance cycling etc.

\section{MARATHON RUNNING INDUCED RESPIRATORY INFECTIONS}

Marathon running is a very long duration and sustained aerobic form of exercise, in general parlance. But, as already indicated, among the elite marathon runners the aerobic part of the metabolic pathway is less dominant during the marathon run, as these elite runners muscle preference seems more towards the anaerobic metabolic pathways as these elite marathon runners would generally run at a high to very high intensities during which the free fatty acid metabolism may not be favored. But still the aerobic metabolism is also simultaneously activated among these elite runners, due to their efficient erythrocytic and mitochondrial apparatuses, which make them to burn even the fats in significant proportions to derive energy for high intensity marathon run. Hence, the possibility of the high levels of oxidative stress is a biggest possibility even among the elite marathon runners both of men and women. Oxidative phosphorylation during the mitochondrial electron transport chain and during the Krebs's cycle, and release of free radical based oxidants is imminent, which might pose threat to the cell membrane integrity and also nuclear membrane integrity ${ }^{15}$ and could cause mutations in the genes through the activation or over expression of transcription factors of the concerned genes or else through the other possible oxidative mechanism.

This oxidative stress mechanism seems more prominent among the less trained and less adapted recreational marathon runners, who participate in various city marathons for the sake of improved health. The trend of participation in marathons by huge number of young and old alike, though a good phenomenon, but in terms of exercise immunology the recreational runners need to be very cautious in their preparation for such arduous events. Those who are participating in the marathon events not on competitive basis may be considered as the recreational marathon runners, and these numbers are growing day by day due to the marketing promotions done by the organizers, as it involves lot of money and financial gain to the organizers. Majority of these recreational runners seems very inactive in their earlier life including during their growing ages and through the adult ages, but they tend to develop sudden spurt of interest in these events and start running training and start participating in these events frequently. It is difficult for all these enthusiastic runners to have the access of scientific training and preparation as there are very small number of qualified exercise trainers and also the possibility of dependence on the internet sources for learning how to train for marathon running events is another bigger drawback. Though some of the enthusiastic running clubs are providing the guidance for their training, the scientific standards of these efforts are questionable in terms of adequacy of knowledge of the trainers, who are mostly the experienced runners. Since, the exercise physiology and exercise endocrinology and exercise nutrition are highly scientific, it would be 
difficult for these runners to have access for very scientific help in their training and preparation for their ultra-endurance efforts.Certainly, most of these recreational runners may be considered as inadequately prepared or else inadequately adapted to take up the high intensity sustained marathon runs as it demands extreme levels of fitness, especially the functional fitness of the whole body. It is also true that several of these recreational runners are so highly motivated suddenly due to the impact and influence of the running groups and their constant interactions, they tend to progress to longer distances very soon without proper consolidation to face the much longer distance running. Since, most of these recreational runners, are also working individuals, the recovery after the training and the competitive run may be compromised, exposing the individuals to the bigger levels of oxidative and inflammatory stress.

Excessive inflammation and oxidative damages to the epithelial tissue of the bronchial tracts of the upper and the lower respiratory tracts could induce for the respiratory tract damages causing the scope for the entry of the pathogens like virus, bacteria etc. and could cause for the mild to severe infections ${ }^{27}$. Moreover, the exercise immunology studies are indicating that the inflammation could cause for changes in the synthesis, production and proliferation of the immune bodies and might alter the immune capacity of the recreational runners. For, example there are studies clearly indicating that severe form of sustained running could lead for the suppression in several immune proteins of mucosal immune system ${ }^{17}$, like immunoglobulins ( $\operatorname{IgA}, \operatorname{IgG}$ etc.), Lactoferrins ${ }^{12,26}$ etc., which are the first like of defense against the intruding pathogens upon the respiratory tract. The high levels of tissue inflammation or even the low levels of chronic inflammation due to improper recovery from training could also cause for severe compromises in $\mathrm{C}$ cell mediated immunity, causing suppressed immunity among the recreational runners either temporarily or for chronically. There were some scientific studies in exercise immunology, that reiterate that the high intensity sustained running efforts are causing severe compromises in bronchial immunity either soon after the event or soon after the severe training program, induces pro active environment for the infections in the upper and lower respiratory tract and especially in the upper respiratory $\operatorname{tract}^{19}$. Severe and frequent upper respiratory tract infections (URTIs) could induce severe debilitating efforts on the respiratory tissues leading to prominent forms of diseases like exercise induced asthma or exercise induced bronchial restrictions and spasms ${ }^{21}$.

\section{OXIDATIVE AND INFLAMMATORY STRESS OF HIGH INTENSITY SUSTAINED RUNNING}

Excessive and uncontrolled oxidative and inflammatory stress could induce conditions of impaired homeostasis, that could cause for proactive environment for the tissue inflammation, tissue damage and other such consequences due to the excessive release of oxidants and inflammatory factors. Unadopted buffer system may be inefficient in terms of the removal of protons in the circulation, produced due to the high intensity running could cause for havoc in the biorhythms of the organism leading to hormonal and enzymatic disturbances. As already indicated earlier, the uncontrolled and excessive release of oxidants like super oxides, nitrites due to probably the mismatch of the antioxidative capacity of the recreational runners who are inadequately adapted may face consequences like threat to the cell membrane integrity and DNA mutations. Oxidative stress during the high intensity sustained aerobic running like marathon running may be very high on the respiratory track epithelial membranes. Apart from the oxidative stress the epithelial membranes and the surrounding tissues of the bronchial tracts, during the high intensity sustained marathon running, individuals are also exposed to the high levels of inflammatory stress ${ }^{20}$.

It is important to understand that both the inflammation and oxidative mechanisms are very natural to the metabolic processes and are in general considered as necessary phenomenon for enhanced protection of the organism, as these two mechanisms in fact initiate for the enhanced antiinflammatory and anti-oxidative capacities among the individuals. Inflammatory stress initiates the release of pro inflammatory cytokines like Interleukin-6, Interleukin-1ra, Tumor Necrosis Factor $\alpha$, PGC $1 \alpha$, and several such cytokines that in turn would evoke for the release of anti-inflammatory cytokines and several other molecules that would tackle the inflammatory damages ${ }^{11}$. In the same way, the oxidative stress also creates such a biological environment, that would induce for the release of endogenous anti-oxidants to neutralize the oxidants release during the oxidative stress of rigorous high intensity exercise like superoxide dismutase (SODs) etc ${ }^{24}$. The stress from the controlled inflammation and oxidative stress also provides the organism an opportunity to understand the 
metabolic sequences to counter these phenomena and would cause for the enhanced immunity, leading to enhanced disease prevention capacity among the individuals. Only the problems lie with the uncontrolled and excessive inflammation and oxidative stress, that may be due to improper adaptation of the recreational runners, or else lack of proper rest and recovery maybe through proper rest, through proper nutritional supplementation etc ${ }^{3,22}$.

\section{UNDERSTANDING URTI SYMPTOMS AMONG THE RECREATIONAL RUNNERS}

Exercise immunologists' perspective of open window theory of infection proneness of respiratory tracts among the ultra-endurance runners has evoked lot of interest among the sportspersons, trainers, coaches and sports scientists ${ }^{19}$. Due to the effect of uncontrolled inflammatory and oxidative stress to the lung tissue during the high intensity sustained aerobic efforts like marathon running and ultraendurance cycling etc ${ }^{25}$. the possibility of the suppression of immune strength ${ }^{9}$ couple with the epithelial inflammation of the lungs could lead for the easy infection to pathogens during such high intensity prolonged physical activity efforts ${ }^{2}$. This might cause for both upper respiratory and lower respiratory infections and may be manifested with the light to severe flu like symptoms. The upper respiratory infections among the endurance runners, especially among the recreational runners seems very important to understand and monitor as the recreational runners seems less adapted for the higher order physical activities ${ }^{6}$. URTIs among the recreational runners could lead for much bigger lung health problems and also could make some chronic health issues among them if not attended to them appropriately and in time. Repeated and chronic exposure to such upper respiratory tract infections are to be avoided for the long standing respiratory health ${ }^{23}$.

Quantification of URTI symptoms seems very essential method of understanding the intensity of the problem and for this there are several methods to adopt. One such method, and very easy to adopt method seems the questionnaire method. A highly credible questionnaire to quantify the intensity of the URTIs was prepared, developed and calibrated through several research projects by the Department of Family Medicine and Public Health of the University of Wisconsin, U.S.A, and is considered as very scientific and credible by scientific community to measure the upper respiratory tract infection status. A total of thirty-two symptoms were identified and were incorporated as thirty two questions in this questionnaire with zero to seven scale options to quantify the symptoms in total. Some of the important symptoms for the URTIs incorporated into the questionnaire are sore throat, running nose, body ache, body ache, sinus symptoms, fever symptoms etc. To understand the effect of the high intensity sustained endurance run like marathon running on the URTI symptoms status, the questionnaire would be administered before the running event and also after the conclusion of the marathon event for few days up to ten days successively to understand the variability of the URTI symptoms scores of the recreational runners and to understand the possible upper respiratory tract infection status among them.

Though the Upper respiratory tract infections seem external in their manifestations through several symptoms as already indicated, it is also ideal and scientific to know the lung function before addressing the lung health further. The science of pulmonology suggests that the lung function need to be understood to monitor the respiratory health of the individual and hence the recreational runners need to monitor not only their upper respiratory infection symptoms status but also their lung function status to keep track of the respiratory health as they involve regularly in high intensity sustained endurance runs during their training as well during their marathon competitions. In case of elite marathon runners, it would be ideal to monitor their lung function along with the URTI symptoms as their running performance might affect significantly. The respiratory tract infections could induce for mucosal secretions and other inflammatory developments causing reductions in the lung capacities and also in the gaseous transfusion capacities ${ }^{4}$. This problem is not just specific only to the elite marathon runners, but also for the recreational runners, as some of the recreational runners may be serious in their competition achievements. Lung function and the respiratory infections are correlated and hence it is also important for the recreational runners to regularly monitor their lung function changes, which may reflect their lung health also ${ }^{14}$. Digital spirometer has been a very effective and credible method of quantifying various lung function capacities of the individuals ${ }^{13}$. Some of the very important pulmonary function variables that would reflect the lung health of the individuals are FEV (Forced Expiratory Volume), $\mathrm{FEV}_{1}$ (Forced Expiratory Volume in the first second of the effort), $\mathrm{FEV}_{1} / \mathrm{FVC}$ ratio (ratio between the Forced Expiratory Volume in the first second and the Forced Vital 
Capacity) ${ }^{7}$. Studies are indicating that the lung values of the individuals may reflect the lung health of the individual and hence the recreational runners may be required to regularly monitor both their respiratory infection status through the symptoms and also through the measurement of respiratory capacities at regular intervals to avoid serious lung infections ${ }^{5,16}$.

\section{CONCLUSION AND RECOMMENDATIONS}

Recreational runners, in general are less adapted to participate in the high intensity very long duration aerobic exercises like marathon running due to various reasons, and hence need more vigorous and scientific training protocols that make them to get gradual exposure to the high intensity long duration running events so that they may not be at risk of upper respiratory tract infections, that may arise due to the uncontrolled oxidative and inflammatory stress that may be prone due to the acute and frequent involvement in the long to very long duration high intensity running events like half and full marathons. The recreational runners are also of different age groups and especially the aged recreational runners need more consolidation of their aerobic fitness before they graduate themselves to run more intense and ultra distance running events. It is also essential for such recreational runners to regularly monitor their respiratory tract infection status through quantitative procedures and also their lung capacities, to correlate their lung health as they increase their long-distance running training and participation.

\section{REFERENCES}

[1] Adam P Lightfoot, and Robert G Cooper. The Role of Myokines in Muscle Health and Disease, CurrOpinRheumatol, 2016 Nov; 28(6):661-6.

[2] Adriele V Souza, Jéssica S Giolo, Renata R Teixeira, Danielle D Vilela et.al. Salivary and Plasmatic Antioxidant Profile Following Continuous, Resistance, and High-Intensity Interval Exercise: Preliminary Study, Oxid Med Cell Longev. 2019 Nov 26; 2019:5425021.

[3] Alessandro Pingitore, Giuseppina Pace Pereira Lima, Francesca Mastorci et.al.Exercise and Oxidative Stress: Potential Effects of Antioxidant Dietary Strategies in Sports, Nutrition. Jul-Aug 2015;31(7-8):916-22.

[4] AndréanneCôté, Julie Turmel, and Louis-Philippe Boulet . Exercise and Asthma, Semin Respir Crit Care Med. 2018 Feb; 39(1):19-28.

[5] Andrew J Simpson, Lee M Romer, and Pascale Kippelen. Self-reported Symptoms After Induced and Inhibited Bronchoconstriction in Athletes, Med Sci Sports Exerc. 2015 Oct;47(10):2005-13.

[6] Antonio Jose Grande, Justin Keogh et.al. Exercise Versus No Exercise for the Occurrence, Severity, and Duration of Acute Respiratory Infections, Cochrane Database Syst Rev. 2020 Apr 4;4(4):CD010596.

[7] Bhumika Aggarwal, AruniMulgirigama, and Norbert Berend. Exercise-induced Bronchoconstriction: Prevalence, Pathophysiology, Patient Impact, Diagnosis and Management, NPJ Prim Care Respir Med. 2018 Aug 14; 28(1):31.

[8] Carol Ewing Garber, Bryan Blissmer, Michael R Deschenes, Barry A Franklin et.al. American College of Sports Medicine Position Stand. Quantity and Quality of Exercise for Developing and Maintaining Cardiorespiratory, Musculoskeletal, and Neuromotor Fitness in Apparently Healthy Adults: Guidance for Prescribing Exercise, Med Sci Sports Exerc. 2011 Jul;43(7):1334-59.

[9] D C Nieman. Special Feature for the Olympics: Effects of Exercise on the Immune System: Exercise Effects on Systemic Immunity, Immunol Cell Biol. 2000 Oct;78(5):496-501.

[10] Darren E R Warburton, and Shannon S D Bredin. Health Benefits of Physical Activity: A Systematic Review of Current Systematic Reviews, CurrOpinCardiol. 2017 Sep;32(5):541-556.

[11] Dorota Kostrzewa-Nowak, Andrzej Ciechanowicz et.al.Damage-Associated Molecular Patterns and ThCell-Related Cytokines Released After Progressive Effort, J Clin Med. 2020 Mar 23;9(3):876.

[12] ElisabetCantó, Emma Roca, Lidia Perea, Ana Rodrigo-Troyano, et.al. Salivary immunity and lower respiratory tract infections in non-elite marathon runners, PLoS One. 2018; 13(11): e0206059.

[13] Enrique Diaz-Guzman, Kevin McCarthy et al. Frequency and Causes of Combined Obstruction and Restriction Identified in Pulmonary Function Tests in Adults, Respir Care 2010;55(3):310 -316.

[14] James H Hull, Les Ansley et.al. Eucapnic Voluntary Hyperpnea: Gold Standard for Diagnosing ExerciseInduced Bronchoconstriction in Athletes? Sports Med. 2016 Aug;46(8):1083-93.

[15] John D Brannan, and James A Turton. The Inflammatory Basis of Exercise-Induced Bronchoconstriction, Phys Sportsmed. 2010 Dec; 38(4):67-73.

[16] John Dickinson, Alison McConnell, and Greg Whyte. Diagnosis of Exercise-Induced Bronchoconstrict ion: Eucapnic Voluntary Hyperpnoea Challenges Identify Previously Undiagnosed Elite Athletes with Exercise-Induced Bronchoconstriction, Br J Sports Med. 2011 Nov; 45(14):1126-31. 
[17] Kay Rutherfurd-Markwick, Carlene Starcket.al. Salivary Diagnostic Markers in Males and Females during Rest and Exercise (cycling exercise of sixty minutes etc). J Int Soc Sports Nutr. 2017 Aug 10; $14: 27$.

[18] Luana G. Leal, Magno A. Lopes and Miguel L. Batista Jr.Physical Exercise-Induced Myokines and Muscle-Adipose Tissue Crosstalk: A Review of Current Knowledge and the Implications for Health and Metabolic Diseases, Front. Physiol., 24 September 2018 https://doi.org/10.3389/fphys.2018.01307.

[19] M W Kakanis, J Peake, E W Brenu, M Simmonds, et.al. The Open Window of Susceptibility to Infection after Acute Exercise in Healthy Young Male Elite Athletes, Exerc Immunol Rev. 2010; 16:119-37.

[20] Mariana Couto, Diana Silva, et.al.Exercise and Airway Injury in Athletes, Acta Med Port. Jan-Feb 2013; 26(1):56-60.

[21] Matteo Bonini, and William Silvers. Exercise-Induced Bronchoconstriction: Background, Prevalence, and Sport Considerations, Immunol Allergy Clin North Am. 2018 May; 38(2):205-214.

[22] Nanci S. Guest, Justine Horne, Shelley M. Vanderhout and Ahmed El-Sohemy. Sport Nutrigenomics: Personalized Nutrition for Athletic Performance, Frontiers in Nutrition, February 2019 | Volume 6 | Article 8. doi: 10.3389/fnut.2019.00008.

[23] Neil P Walsh. Recommendations to Maintain Immune Health in Athletes, Eur J Sport Sci. 2018 Jul; 18(6):820-831.

[24] O. F. Araneda, T. Carbonell, and M. Tuesta. Update on the Mechanisms of Pulmonary Inflammation and Oxidative Imbalance Induced by Exercise, Oxid Med Cell Longev. 2016; 2016: 4868536.

[25] Rajasekhar Kali Venkata, Acute cycling sport causes upper respiratory tract infections and lung function loss among male recreational cyclists of different age groups. Journal of Science \& cycling, 2014, 3(2).

[26] S K Gill, A M Teixeira, F Rosado, J Hankey et.al, The Impact of a 24-h Ultra-Marathon on Salivary Antimicrobial Protein Responses. Int J Sports Med. 2014 Oct; 35(11):966-71.

[27] Vernon Neville, Michael Gleeson, and Jonathan P Folland.Salivary IgA as a Risk Factor for Upper Respiratory Infections in Elite Professional Athletes, Med Sci Sports Exerc. 2008 Jul; 40(7):1228-36.

Citation: Mr. RajendarKommu. "Upper Respiratory Tract Infection Status Symptoms among the Recreational Endurance Runners- A Perspective" International Journal of Sports and Physical Education (IJSPE), vol 6, no. 4, 2020, pp. 01-06. doi: https://doi.org/10.20431/2454-6380.0604001.

Copyright: (C) 2020 Authors. This is an open-access article distributed under the terms of the Creative Commons Attribution License, which permits unrestricted use, distribution, and reproduction in any medium, provided the original author and source are credited. 